same correlation. It would be rational to highlight only one zone of prognosis before and after 5,8 .

Disclosure of Interests: None declared.

DOI: 10.1136/annrheumdis-2021-eular.3627

\section{AB0456 HYDROXYCHLOROQUINE MIGHT REDUCE MORTALITY IN PATIENTS WITH SYSTEMIC SCLEROSIS}

M. Ozmen ${ }^{1}$, E. Otman Akat ${ }^{1}$, S. Gucenmez ${ }^{1}$, G. Kabadayi ${ }^{1}$, E. Durak Ediboglu ${ }^{1}$, G. Alp ${ }^{1}$, H. Cinakli ${ }^{1}$, E. Erpek ${ }^{1}$, I. Kurut Aysin ${ }^{1}$, O. Bayindir ${ }^{1}$, D. Solmaz ${ }^{1}$ S. Akar. ${ }^{1}{ }^{1}$ Izmir Katip Celebi University Faculty of Medicine, Rheumatology, Izmir, Turkey

Background: Systemic sclerosis (SSc) is a devastating disease that has a profound impact on life expectancy, reflected by a standardised mortality ratio of 3,5. There is still limited data regarding the predictive factors for mortality in patients with SSc. Determining those factors could guide in disease management and follow up. ${ }^{1}$

Objectives: We aimed to identify the predictive factors for death in SSc.

Methods: Patients followed in a tertiary rheumatology clinic in the last 5 years were included in this retrospective study. All of the patients met the ACR / EULAR SSc 2013 criteria. Medical records of the patients were reviewed. Follow up time was defined as the time period from the first admission of the patient to our rheumatology clinic until the date of death or the date on which the study was performed. Candidate predictive factors for mortality were tested by Kaplan-Meier (with Log rank) and Cox-regression analyses.

Results: In total 146 patients (mean age $55.6 \pm 12.3$ years, female $89.7 \%$, diffuse cutaneous type SSc $45.2 \%$ ) were included in the study (Table 1). The mean age at diagnosis of study group was $48 \pm 13.7$ years. The median duration of follow up was 71 (6-228) months. Fourteen (10\%) patients died during follow-up. The causes of death were: pulmonary (7), renal (2) and cardiac diseases (1), infection (3) and cancer (1).

Univariate analysis revealed that age at diagnosis $(p=0.028)$, SSc subtype $(p=0.035)$, the presence of interstitial lung disease $(p=0.002)$, oesophageal involvement (on computed tomography) $(p=0.030)$, pulmonary artery systolic pressure of $\geq 35 \mathrm{mmHg}$ (measured by transthoracic echocardiography) $(p=0.004)$, glucocorticoid $(p=0.029)$, hydroxychloroquine $(p=0.002)$ and cyclophosphamide $(\mathrm{p}=0.006)$ usage at any time were associated with mortality (Figure 1). Multivariate analyses model formed with age at diagnosis (B: 0.055 , $95 \% \mathrm{Cl}, 1.005-1.112 ; \mathrm{p}=0.033$ ), SSc subtype (B: $0.963,95 \% \mathrm{Cl} 0.541-12.684$; $\mathrm{p}=0.231$ ), glucocorticoid ( $\mathrm{B}: 1.396,95 \% \mathrm{Cl}, 0.487-33.507 ; \mathrm{p}=0.196)$ and hydroxychloroquine usage ( $\mathrm{B}:-1.50,95 \% \mathrm{Cl}, 0.061-0.816 ; \mathrm{p}=0.023$ ) showed that age at diagnosis and hydroxychloroquine usage were independent predictive factors for mortality in patients with SSc.

Conclusion: The results of the study revealed for the first time that apart from the age at diagnosis hydroxychloroquine might reduce mortality in patients with SSc. Further studies are needed to prove of this information.

REFERENCES:

[1] Elhai $M$, et al. Ann Rheum Dis 2017;0:1-9. doi:10.1136/ annrheumdis-2017-211448

Table 1. The demographic and clinical features in patients with systemic sclerosis.

\begin{tabular}{lc}
\hline Characteristic & Baseline \\
\hline Age at diagnosis & $48 \pm 13.7$ \\
Female sex, $\mathbf{n}(\%)$ & $131(89.7)$ \\
Duration of follow-up, months ${ }^{* *}$ & $71(6-228)$ \\
Disease subtype, $\mathbf{n}(\%)$ & \\
Diffuse / Limited & $66(45.2) / 80(54.8)$ \\
Autoantibodies, $\mathbf{n}(\%)$ & \\
Anti-Scl70 antibody & $50 / 143(35.0)$ \\
Anti-Centromere antibody & $62 / 143(43.4)$ \\
Immunsuppresive medication, ever, $\mathbf{n}(\%)$ & \\
Hydroxychloroquine & $91 / 143(63.6)$ \\
Mycophenolate mofetil & $18 / 145(12.4)$ \\
Azathioprine & $47 / 145(32.4)$ \\
Cyclophosphamide & $24 / 145(16.6)$ \\
Glucocorticoid & $80 / 140(57.1)$ \\
Others, $\mathbf{n}$ (\%) & \\
ILD & $68 / 130(52.3)$ \\
Pericardial effusion, ever & $26 / 133(19.5)$ \\
Esophageal dilation (detected by CT) & $51 / 128(39.8)$ \\
sPAP $\geq 35 m m H g$, ever (measured by ECHO) & $46 / 142(32.4)$
\end{tabular}

"Parameter presented as mean \pm SD"Parameter presented as median (min-max)CT, computed tomography; ECHO, echocardiogram; ILD, interstitial lung disease; SPAP, systolic pulmonary artery pressure

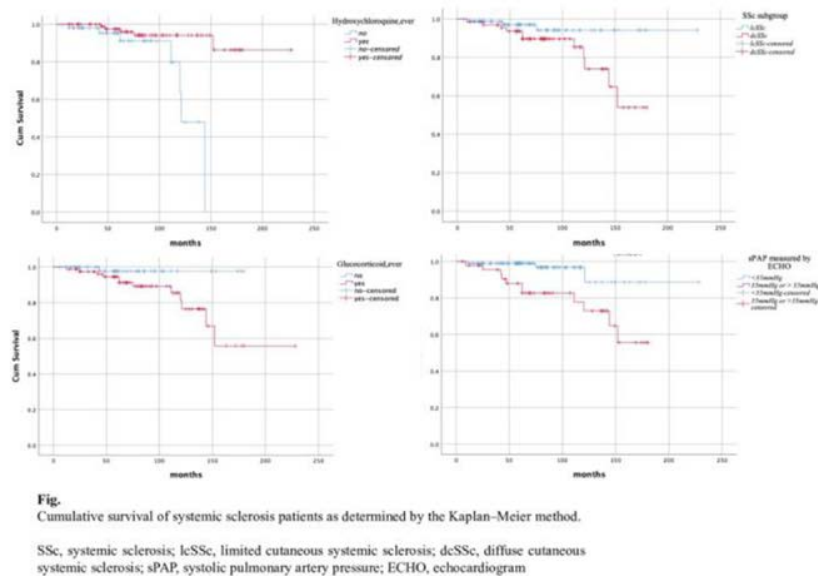

Figure 1.

Disclosure of Interests: None declared.

DOI: 10.1136/annrheumdis-2021-eular.3685

\section{AB0457 HIPPOCAMPAL SUBFIELDS VOLUMES REDUCTION IN PATIENTS WITH SYSTEMIC SCLEROSIS: A LONGITUDINAL MAGNETIC RESONANCE IMAGING (MRI) VOLUMETRIC STUDY}

D. Pereira ${ }^{1}$, M. Freschi ${ }^{1}$, R. Frittoli ${ }^{1}$, A. C. Londe ${ }^{1}$, T. Amaral $^{2}$, S. Dertkigil ${ }^{3}$, A. P. Del Rio ${ }^{4}$, F. Cendes ${ }^{5}$, L. Rittner ${ }^{6}$, S. Appenzeller ${ }^{4} .{ }^{1}$ UNICAMP, Pathophysiology Graduate Program, Campinas, Brazil; ${ }^{2}$ UNICAMP, Medicine, Campinas, Brazil; ${ }^{3}$ UNICAMP, Radiology, Campinas, Brazil; ${ }^{4}$ UNICAMP, Orthopedics, Rheumatology and Traumatology, Campinas, Brazil; ${ }^{5}$ UNICAMP, Neurology, Campinas, Brazil; ${ }^{6}$ UNICAMP, Electrical Engeneering, Campinas, Brazil

Background: Systemic sclerosis (SSc) is a multisystem disease characterized by functional and structural abnormalities of small blood vessels, fibrosis of the skin and internal organs. In addition, volume reduction and shape abnormality of the hippocampus have been demonstrated in rheumatic and neurodegenerative diseases. However, the neuroanatomy of the hippocampus is complex and is not a uniform structure, consisting of subfields with distinct morphology: cornu ammonis (CA) subfields CA1-4, dentate gyrus (DG), fimbria, and adjacent subiculum and presubiculum [1].

Objectives: To investigate the hippocampal subfields volumes reduction in SSc patients using MRI

Methods: In this study we included 37 SSc patients (33 women, mean age of $53.46, S D \pm 12.29$; range $30-78$ ) and 37 healthy controls $(\mathrm{HC})$ ( 31 women, mean age of $48.41, S D \pm 12.20$; range $29-80)$. Cognitive evaluation was performed using the Montreal Cognitive Assessment (MoCA), mood disorders were determined through Beck's Depression (BDI) and Beck's Anxiety Inventories (BAI). SSc patients were further assessed for clinical and laboratory SSc manifestations, disease activity (Valentini Activity Index), severity activity (Medsger Severity Index). MRI protocol consisted of: Sagittal T1-weighted images performed with a Philips 3.0T MRI scanner. MRI brain structure volumetric was done through volBrain [2]. After 48 months MRI acquisition were repeated in 26 SSc patients and 12 healthy volunteers. Statistics was performed according nature of the variable.

Results: We observed a significant reduction in hippocampal subfields volumes in SSc patients when compared to controls: Total hippocampi (SSc: mean volume = $4.78 \mathrm{~cm}^{3} ; \mathrm{SD}=0.38 ; \mathrm{HC}$ : mean volume $=5.01 \mathrm{~cm}^{3} ; \mathrm{SD}=0.38 ; \mathrm{p}=0.033$ ). Reduction in volume of the total hippocampi was associated with Raynaud's phenomenon $(p=0.006)$. A longitudinal study showed a reduction in volume of the hippocampus subfields volumes when compared to patient's baseline: Total hippocampi (mean initial volume $=4.78 \mathrm{~cm}^{3} ;$ mean follow-up volume $=4.50 \mathrm{~cm}^{3}, \mathrm{p}=0.027$ ); Total CA1 (mean initial volume $=1.59 \mathrm{~cm}^{3}$; mean follow-up volume $=1.58 \mathrm{~cm}^{3}, p<0.0001$ ); Reduction in volume of the total hippocampi was associated with presence of current use of prednisone $(p=0.008)$. Reduction in left CA1 left associated with current use of prednisone $(p=0.014)$. Reduction in total subiculum was associated with presence of calcinosis $(p=0.023)$. No significant changes were observed in hippocampal subfields volumes in controls over the follow-up period.

Conclusion: This study provides evidence of hippocampus subfields volumes reductions in SSc patients when compared to controls and was associated with 
Raynaud's phenomenon, current use of losartan and correlated with BAI scores. Also, a longitudinal study showed a reduction in volume of the hippocampus subfields volumes when compared to patient's baseline associated with calcinosis and current use of prednisone.

REFERENCES:

[1] Eichenbaum, Howard. "A cortical-hippocampal system for declarative memory." Nature Reviews Neuroscience 1.1 (2000): 41-50.

[2] Manjon JV, Coupe P (2016) volBrain: an online MRI brain volumetry system. Front Neuroinform 10:30.

Disclosure of Interests: None declared.

DOI: 10.1136/annrheumdis-2021-eular.3815

\section{AB0458 INTERSTITIAL LUNG DISEASE: OBSERVATION DATA FROM A MALAYSIAN TERTIARY CENTRE}

J. Raja ${ }^{1}$, S. Muthusamy ${ }^{1}$, C. M. $\mathrm{Ng}^{2} .{ }^{1}$ University of Malaya, Division of Rheumatology, Department and Faculty of Medicine, Kuala Lumpur, Malaysia; ${ }^{2}$ University of Malaya, Institute of Mathematical Sciences, Faculty of Science, Kuala Lumpur, Malaysia

Background: Interstitial lung disease (ILD) is the leading cause of death in scleroderma (SSc) with decline in FVC as a predictor of mortality in patients with SSc-ILD, especially in the early course of the disease.

Objectives: The aim of this study is to determine the survival rate of SSc-ILD in a Malaysian cohort of patients from University Malaya Medical Centre (UMMC). Methods: 61 patients clinically diagnosed with SSc-ILD were identified and prospectively recruited. Baseline demographic data were collected. Kaplan-Meier analysis was used to estimate the survival.

Results: Females were predominant (56, 91.8\%). 39 (64\%) had limited cutaneous SSc. Majority were ethnicity Chinese 30 (49.2\%), followed by Malays 20 (32.8\%), Indians 7 (11.4\%) and others 4 (6.6\%). Mean age was 56.25 (SD \pm $12.5)$ years while mean duration of disease (non-Raynaud's disease onset) was 10.5 years $(S D \pm 9.2)$ (range of 1 year to 44 years) years. $29(47.5 \%)$ patients were positive for anti-ScL-70, whereas $6(9.8 \%)$ patients were anti-centromere positive. There were $16(26.2 \%)$ deaths. Median survival was 24 years. Patients had a sharper drop in survival probability for the first 10 years compared to the next 20 years (Figure 1). Median survival in limited subset was 24 years whereas in diffuse subset was 11 years. Patients from the limited subset appeared to have higher chance of surviving for 10 years and above, compared to those in the diffuse subset (Figure 2).

Conclusion: The results demonstrate the poor survival in SSc-ILD patients. The survival rate tends to be worse in the first 10 years of SSc disease duration. Survival rate was poorer in patients with diffuse cutaneous subset.

REFERENCES:

[1] Parelas A et al. Lancet Resp Med 2020

[2] Goh NS et al. Am J Respir Crit Care Med 2008

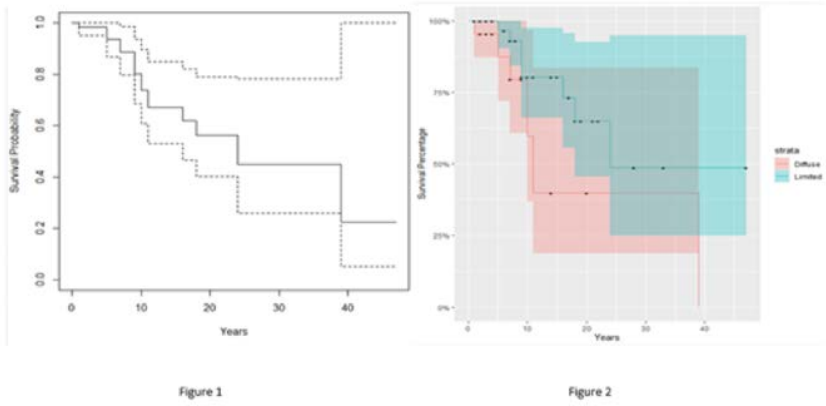

Disclosure of Interests: JASMIN RAJA Speakers bureau: For Boehringer Ingelheim for topic on Scleroderma-ILD, Grant/research support from: From Boehringer Ingelheim for scleroderma research, Shantini Muthusamy: None declared., CHOUNG MIN NG: None declared.

DOI: 10.1136/annrheumdis-2021-eular.3830

\section{AB0459 $\quad$ ARTICULAR INVOLVEMENT IN PATIENTS WITH SYSTEMIC SCLEROSIS}

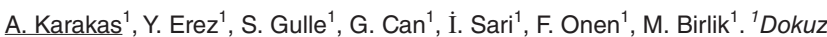
Eylul University Faculty of Medicine, Rheumatology, Izmir, Turkey

Background: SSc (systemic sclerosis) is a connective tissue disease characterized by small vessel vasculopathy, production of autoantibodies, and fibroblast dysfunction leading to increased deposition of extracellular matrix in the skin and internal organs mainly. Therewithal, many SSc patients develop musculoskeletal symptoms during the course of their illness. Different rheumatic complaints such as arthralgia, arthritis, contractures, tendon friction rubs, calcinosis, and acroosteolysis can be seen as musculoskeletal symptoms in SSc patients [1]

Objectives: To provide an overview of the spectrum of articular involvemen in SSc and determine the relationship between these involvements and Rheumatoid factor (RF) and Anti-cyclic citrullinated peptide (Anti-CCP) positivity and organ involvements.

Methods: We performed a retrospective cohort study involving 232 SSc patients who were followed up in our department of rheumatology between 2000 and 2020 years. The patients were divided into two groups as limited and diffuse SSc. Age, gender, weight, height, smoking habits, duration of illness, follow-up duration, other systemic organ involvement, and radiographic findings were recorded. Diagnostic tests such as RF, Anti-CCP, ANA, ENA panel tests, direct radiographs were examined.

Results: The mean age of the patients was $59.9 \pm 12.8$ and $88.4 \%$ of them were women. $69.3 \%$ of patients were limited SSc. At any stage of the disease, $39.1 \%$ of all patients had arthralgia and $34.1 \%$ had arthritis. The arthritis rate was similar between the SSc groups $(p=0.396)$. RF and anti-CCP positivity rates were similar between the SSc groups. Although RF and anti-CCP positivity rates were higher in the patient group with arthritis, it was not statistically significant (respectively $p=0.563, p=0.072$ ). Interestingly, the lung involvement rate was higher in patients with arthritis $(63.3 \%$ versus $46.4 \%)(p=0.015)$. Other clinical, demographic characteristics, laboratory, and radiographic findings of the patients are shown in Table 1.

Conclusion: Articular involvement in SSc is a common clinical feature seen in one-third of patients regardless of the type of disease. Although RF and AntiCCP positivity are more common in patients with arthritis, it was not statistically significant. Interestingly, arthritis is a more common manifestation in patients with lung involvement.

\section{REFERENCES:}

[1] R. D. Sandler, M. Matucci-Cerinic, and M. Hughes, "Musculoskeletal hand involvement in systemic sclerosis," Seminars in Arthritis and Rheumatism, vol. 50, no. 2. 2020, doi: 10.1016/j.semarthrit.2019.11.003.

Table 1. Demographic and clinical characteristics of b/tsDMARDs patients

Limited SSc Diffuse SSc All patients

\begin{tabular}{|c|c|c|c|c|}
\hline Table-1 & $(n=161)$ & $(n=71)$ & $(n=232)$ & $\mathbf{P}$ \\
\hline Age (years) (Mean $\pm S D)$ & $60.5 \pm 12.7$ & $58.6 \pm 12.9$ & $59.9 \pm 12,8$ & 0.295 \\
\hline Disease duration (years) (Mean $\pm S D$ ) & $11.5 \pm 6.6$ & $12.4 \pm 8.1$ & $11.8 \pm 7,1$ & 0.384 \\
\hline Weight (kg) (Mean \pm SD) & $68.2 \pm 13.3$ & $66.2 \pm 15.6$ & $67.6 \pm 14.1$ & 0.331 \\
\hline BMI $\left(\mathrm{kg} / \mathrm{m}^{2}\right)($ Mean $\pm \mathrm{SD})$ & $27.6 \pm 5.5$ & $26.2 \pm 6.1$ & $27.1 \pm 5.7$ & 0.102 \\
\hline Female, n (\%) & $146(90.7)$ & $59(83.1$ & $205(88.4)$ & 0.097 \\
\hline Current and ex smoker, $\mathrm{n}(\%)$ & $60(37.2)$ & $18(25.4)$ & $78(33.6)$ & 0.077 \\
\hline Digital ulcer, n (\%) & $53(32.9)$ & $34(47.9)$ & 87 (37.5) & $0.03^{\star}$ \\
\hline Contracture on hand & $28(17.4)$ & $28(39.4)$ & $56(24.1)$ & $<0,001^{*}$ \\
\hline Arthralgia, n (\%) & $63(3$ & $29(31.5)$ & $92(39.7)$ & 0.806 \\
\hline Arthritis, n (\%) & $52(32.3)$ & $27(38)$ & $79(34.1)$ & 0.396 \\
\hline Joint space narrowing on X-ray, $n$ (\%) & $44(51.2)$ & $30(63.8)$ & $74(55.6)$ & 0.160 \\
\hline Joint erosion on X-ray, $\mathbf{n}(\%)$ & 17 (19.8) & $14(29.8)$ & 31 (23.3) & 0.205 \\
\hline Acroosteolysis on X-ray, $\mathrm{n}(\%)$ & $9(10.5)$ & $16(34)$ & $25(18.8)$ & $0.001^{*}$ \\
\hline ANA positivity, $n(\%)$ & $155(96.3)$ & 69 (97.2) & $224(96.6)$ & 0.728 \\
\hline Anti-Scl positivity, n (\%) & $41(28.7)$ & $48(71.6)$ & $89(42.4)$ & $<0.001^{*}$ \\
\hline Anti-centromere positivity, n (\%) & $65(45.5)$ & $10(14.9)$ & $75(35.7)$ & $<0.001^{*}$ \\
\hline RF positivity, n (\%) & $27(17.5)$ & $14(20.6)$ & $41(18.5)$ & 0.589 \\
\hline Anti-CCP positivity, $n(\%)$ & $16(12.7)$ & $7(11.3)$ & 23 (12.2) & 0.782 \\
\hline
\end{tabular}

$\mathrm{P}^{*}$ Independent Samples t Test, Pearson Chi-Square Test, BMI; Body mass index, ANA; Ant nuclear antibody,RF; Rheumatoid factor, Anti-CCP;Anti- Cyclic citrullinated peptide

Disclosure of Interests: None declared.

DOI: 10.1136/annrheumdis-2021-eular.4103

\section{AB0460 \\ THE UTILITY OF USB CAPILLAROSCOPE FOR ASSESSMENT OF RAYNAUD'S PHENOMENON PATIENTS}

S. Lambova ${ }^{1} .{ }^{1}$ Medical University - Plovdiv, Department of propaedeutics of Internal Diseases, Plovdiv, Bulgaria

Background: Videocapillaroscopy is the gold standard for evaluation of nailfold capillaries and the major tool used for differentiation of primary and secondary Raynaud's phenomenon (RP) in rheumatology practice. However, nowadays, there are also accessible alternatives such as USB capillaroscopes, which offer the opportunity to apply capillaroscopic examination at a significantly lower price. Objectives: The aim of the current study was to study the utility of USB capillaroscope (Dinolite) via assessment of capillaroscopic images obtained by patients with primary and secondary RP in rheumatic diseases. 